\title{
The IMF of open star clusters with Tycho-2^
}

\author{
J. Sanner and M. Geffert \\ Sternwarte der Universität Bonn, Auf dem Hügel 71, 53121 Bonn, Germany \\ Received 14 September 2000 / Accepted 7 February 2001

\begin{abstract}
We studied the fields of nine nearby open star clusters based on the Tycho-2 catalogue. We determined membership probabilities for the stars in the cluster fields from the stellar proper motions and used the Tycho- 2 photometry to compute the initial mass function (IMF) of the clusters from the main sequence turn-off point down to approx. $1 M_{\odot}$. We found IMF slopes ranging from $\Gamma=-0.69$ down to $\Gamma=-2.27$ (when the Salpeter 1955 value would be $\Gamma=-1.35$ ). We also studied the membership of individual stars of special astrophysical interest. In some cases previous results had to be revised. As a by-product, we investigated some general properties of the Tycho-2 catalogue; we confirmed that the Tycho-2 proper motions show only marginal deviations from the Hipparcos catalogue. On the other hand, in some regions the completeness of the catalogue seems to decrease at magnitudes brighter than claimed by $\mathrm{H} \varnothing \mathrm{g}$ et al. (2000).
\end{abstract}

Key words. open clusters and associations: general - catalogs - astrometry - stars: kinematics - HertzsprungRussell (HR) and C-M diagrams - stars: luminosity function, mass function

\section{Introduction}

The Tycho-2 catalogue (Høg et al. 2000) provides positions and stellar proper motions in conjunction with two colour photometric data. These were computed from the original Tycho (star mapper) data obtained during the Hipparcos mission (ESA 1997) and ground based first epoch data, including the Astrographic Catalogue (Wood 1970). This work was triggered by the findings of Makarov et al. (2000) who recently found that the precision of the Tycho-2 proper motions are of the same order as the Hipparcos data. With its significantly larger number of stars, the Tycho-2 catalogue therefore is a suitable source of data for studies of stellar kinematics. In earlier investigations Hipparcos data were used for a search for open clusters (Platais et al. 1998) and checks of open cluster candidates (Baumgardt 1998).

In this investigation we used fields of nine nearby open star clusters. We separated the field stars from the cluster members with the help of the proper motions provided by Tycho-2 and determined colour magnitude diagrams (CMDs) from its photometry. From the CMDs we derived the age, distance modulus, and reddening by fitting isochrones and compared the results with, e.g., distances derived from Hipparcos parallaxes (van Leeuwen 1999a, hereafter referred to as vL99a). In a final step, we computed the initial mass function (IMF) of the clusters with

Send offprint requests to: J. Sanner, e-mail: jsanner@astro.uni-bonn.de

* Based on observations of the ESA Hipparcos satellite. a maximum likelihood method. The results of this part of the study can give us clues about the completeness of the Tycho-2 data.

Especially for open clusters, where the contamination of the data with field stars poses a major challenge, knowledge of membership is essential to derive the IMF. The most precise method to determine membership is by studies of stellar kinematics, as one can assume that all cluster stars move in the same way, whereas the distribution of the motions for the field stars is much wider and differently centred. From this point of view, a catalogue like Tycho2 , including photometry and proper motions, provides an ideal opportunity for an IMF study.

The shape of the IMF is an important parameter to understand the fragmentation of molecular clouds and therefore the formation and development of stellar systems. Besides studies of the Solar neighbourhood (Salpeter 1955; Tsujimoto et al. 1997), work on star clusters plays an important role (Scalo 1986, 1998), as age, metallicity, and distance of all cluster stars can be assumed to be the same.

Restricted to certain mass intervals, most studies agree that the IMF can be described by a power law in the form

$\mathrm{d} \log N(m) \sim m^{\Gamma} \mathrm{d} \log m$.

In this notation, the "classical" value found by Salpeter (1955) for the Solar neighbourhood would be $\Gamma=-1.35$. We will not be able to reach smaller masses than about $1 M_{\odot}$ in this study, so that, according to the review of Scalo (1998), our values for $\Gamma$ are expected to be within the 
interval $[1.2 ; 2.2]$, a range which is valid for intermediate mass stars of $1 M_{\odot} \lesssim m \lesssim 10 M_{\odot}$. In particular, the IMF can be expected to show a behaviour which is in accordance with a single power law in this mass range.

Up to now, thorough IMF studies are available only for a few of the nearby star clusters, e.g. the Pleiades (van Leeuwen 1980; Meusinger et al. 1996; Hambly et al. 1999) or Praesepe (Williams et al. 1995; Pinfield et al. 1999). Some of the clusters were also studied by Tarrab (1982), but her work was based on an inhomogeneous data sample with sometimes weak information on membership. Discrepancies of her results with later work have been found (see, e.g., Sanner et al. 2000 for NGC 1960 or Credner 1996 for NGC 2323), so that revisiting the clusters studied by Tarrab (1982) is promising.

Nowadays, although CCD cameras provide a good tool for IMF studies down to low masses $\left(m \ll M_{\odot}\right)$, the limited field size of typical imagers of the order of arc minutes makes comprehensive studies a difficult task: Nearby clusters have a large angular diameter in the sky, so that mosaics of lots of different fields would have to be observed to obtain a general overview of the stellar content of a cluster. For the determination of a cluster IMF a snapshot of one or only few fields is not enough, since spatial inhomogeneities (mass segregation; see, e.g., Raboud \& Mermilliod 1998) are likely to falsify the results. With a uniform photometric and astrometric all sky survey, this goal can be achieved at least down to a reasonable mass limit.

Section 2 contains a description of the data and methods used for this work as well as some general results, followed in Sect. 3 by the results sorted by cluster. The final section contains a summary and conclusions.

\section{Data, data analysis and general results}

Since the Tycho-2 data are claimed to be complete down to $11 \mathrm{mag}$ (see Høg et al. 2000 and the discussion below), we were restricted to a small magnitude (and hence mass) interval. To reach stars with masses as low as possible we chose to study nearby $\left((m-M)_{0} \lesssim 7.5 \mathrm{mag}\right.$ or $\left.d \lesssim 315 \mathrm{pc}\right)$ open star clusters only.

Robichon et al. (1999) published a list of astrometric data of nearby open clusters based on the Hipparcos catalogue. From this information the nine targets listed in Table 1 were chosen. A main criterion for the selection was a clear separation between the field and cluster proper motion centres to make the membership determination an easy task and to avoid the presence of numerous field stars with the same proper motion as the cluster. A maximum of 10 to 15 stars can be expected to belong to the field but to have (within the errors) the same proper motion as the corresponding cluster. The Hipparcos parallaxes of seven of them were recently studied by vL99a to derive the distances of the clusters. Similar computations can also be found in Robichon et al. (1999). Due to the very high quality of the Hipparcos data and the reliability of the results, we preferred these values for the distance moduli
Table 1. List of the open star clusters studied in this work together with their coordinates. For data on the proper motions, see Table 2

\begin{tabular}{lcc}
\hline cluster & $\begin{array}{c}\alpha_{2000} \\
{[\mathrm{~h} \mathrm{~m}]}\end{array}$ & $\begin{array}{c}\delta_{2000} \\
{\left[{ }^{\prime}{ }^{\prime}\right]}\end{array}$ \\
\hline Blanco 1 & 0004.3 & -2956 \\
Stock 2 & 0215.0 & +5916 \\
$\alpha$ Per & 0322.0 & +4837 \\
Pleiades & 0347.0 & +2407 \\
NGC 2451 A & 0745.4 & -3758 \\
IC 2391 & 0840.2 & -5304 \\
Praesepe & 0840.4 & +1940 \\
IC 2602 & 1043.2 & -6424 \\
NGC 7092 & 2132.2 & +4826 \\
\hline
\end{tabular}

and reddenings of these clusters compared with those of previous studies. The results of vL99a and Robichon et al. (1999) agree well within the errors for our clusters, except for Blanco 1 with a deviation of $\Delta(m-M)_{0} \approx 0.3 \mathrm{mag}$. We will discuss this point later (Sect. 3.5).

The Tycho project made use of its own photometric system, referred to as $B_{T}$ and $V_{T}$. These two filters are close to Johnson $B$ and $V$, respectively, and ESA (1997) provides transformation equations to the Johnson system:

$$
\begin{aligned}
V & =V_{T}-0.090 \cdot(B-V)_{T} \\
B-V & =0.850 \cdot(B-V)_{T} .
\end{aligned}
$$

For our purposes, it was not necessary to transform the magnitudes to $B$ and $V$ : The $V$ magnitudes are influenced by errors of up to $\Delta V=0.09 \mathrm{mag}$ - a value which is valid for the redmost stars (i.e. $(B-V)_{T} \approx 1 \mathrm{mag}$ ). The colours can be influenced by up to $\Delta(B-V)=0.15 \mathrm{mag}$, however, this figure again is valid for very red stars only. Since the colours are only required for the isochrone fitting, which is performed on the base of the bright main sequence stars with colours of around $(B-V)_{T}=0$, the deviations from the Tycho to the Johnson system can be disregarded. We will see later that the Tycho-2 $\left(B_{T}, V_{T}\right)$ data lead to lower reddenings than several previous studies of the same objects. This cannot be explained by not transforming the magnitudes to the Johnson system: In addition to the arguments mentioned before, the Johnson colours for stars with $(B-V)_{T} \geq 0$ are bluer than the corresponding Tycho values according to Eq. (3). This is in contrast with the deviating values, which would all lead to a higher reddening.

After extracting sufficiently large areas around the centres of the targets - taking at least twice the diameter given in the Lyngå (1987) catalogue to avoid a biasing due to mass segregation - we derived the membership probabilities $P$ of the individual stars from their proper motions. We fitted a combination of a sharp (for the members) and a wider (for the field stars) two-dimensional Gaussian distribution to the vector point plot diagram. The optimal parameters (location of the field and cluster proper motion centres in the diagram and the standard deviations 
Table 2. List of the open star clusters studied. We give the centres of the proper motions of the field and cluster stars. Since the field proper motions were fitted by a two dimensional Gaussian function, we give the semi major and minor axes $a$ and $b$ and the rotation angle $\phi$ of the $1 \sigma$ ellipse. Furthermore, we add the ratio $r$ of members to all stars in the studied fields. For a comparison of our Tycho-2 proper motion centres with the Hipparcos-based ones from Robichon et al. (1999), see Sect. 2

\begin{tabular}{|c|c|c|c|c|c|c|c|c|c|}
\hline \multirow[b]{2}{*}{ cluster } & \multicolumn{3}{|c|}{ cluster } & \multicolumn{5}{|c|}{ field } & \multirow[b]{2}{*}{$\begin{array}{c}r \\
{[\%]}\end{array}$} \\
\hline & $\begin{array}{c}\mu_{\alpha} \cos \delta \\
{\left[\mathrm{mas} \mathrm{yr}^{-1}\right]}\end{array}$ & 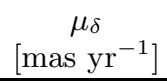 & {$\left[\begin{array}{c}\sigma \\
{\left[\mathrm{mas} \mathrm{yr}^{-1}\right]}\end{array}\right.$} & $\begin{array}{c}\mu_{\alpha} \cos \delta \\
{\left[\mathrm{mas} \mathrm{yr}^{-1}\right]}\end{array}$ & $\begin{array}{c}\mu_{\delta} \\
{\left[\operatorname{mas~yr}^{-1}\right]}\end{array}$ & $\begin{array}{c}a \\
{\left[\mathrm{mas} \mathrm{yr}^{-1}\right]}\end{array}$ & $\begin{array}{c}b \\
{\left[\mathrm{mas} \mathrm{yr}^{-1}\right]}\end{array}$ & $\begin{array}{c}\phi \\
{[\mathrm{deg}]}\end{array}$ & \\
\hline Blanco 1 & +19.27 & +2.81 & 2.02 & +10.98 & -7.71 & 16.48 & 11.93 & +24.5 & 11.1 \\
\hline Stock 2 & +17.15 & -12.94 & 4.61 & +0.36 & -1.58 & 6.76 & 6.40 & -31.8 & 14.0 \\
\hline$\alpha$ Per & +22.63 & -25.74 & 1.52 & -0.74 & -3.53 & 13.11 & 8.14 & -37.0 & 3.7 \\
\hline Pleiades & +19.96 & -45.09 & 1.78 & +5.43 & -11.42 & 17.62 & 14.31 & -53.1 & 7.6 \\
\hline NGC $2451 \mathrm{~A}$ & -21.96 & +14.59 & 1.17 & -5.40 & +4.59 & 11.21 & 7.33 & -57.8 & 5.4 \\
\hline IC 2391 & -24.64 & +23.25 & 1.13 & -6.82 & +6.29 & 12.34 & 7.28 & -48.3 & 6.0 \\
\hline Praesepe & -35.88 & -13.18 & 1.93 & -6.40 & -9.20 & 11.47 & 11.32 & +6.5 & 10.6 \\
\hline IC 2602 & -17.63 & +10.30 & 1.50 & -8.55 & +2.43 & 9.62 & 5.68 & -26.6 & 4.5 \\
\hline NGC 7092 & -8.34 & -19.16 & 1.19 & -2.00 & -2.26 & 10.93 & 6.52 & +41.1 & 2.7 \\
\hline
\end{tabular}

of the two Gaussians) were determined with a maximum likelihood procedure as described in Sanders (1971).

Due to the large fields, the vector point plot diagrams were dominated by the field stars. For some of the clusters, this caused the software to misinterpret the field stars as the cluster members and vice versa. Since the stars determined as members are located within a circle or an ellipse, and the non-members consist of all the rest, the correct membership probabilities could not be computed as the complement of the determined ones: Any star at a sufficient distance from the field centre would obtain a high probability with this method, even if that star is located at the opposite end of the vector point plot diagram, compared with the actual cluster. This problem could be solved by removing all stars with a very large deviation

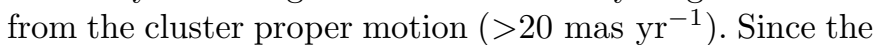
clusters were easily detectable in the vector point plot diagrams and the Robichon et al. (1999) data gave additional clues about their location, it can be excluded that the region covering the target was accidentally eliminated. Also, cross checks of the positions of the stars selected as members in the vector point plot diagram showed that the membership determination was successful.

The vector point plot diagrams (see the diagram of the $\alpha$ Per cluster derived from the Tycho-2 data in Fig. 7 as an example) showed a standard deviation of the cluster stars in the region of $1.5-2$ mas $_{\mathrm{yr}^{-1}}$. Since these values correspond to proper motion errors rather than to internal motions, a good coincidence between our results and the values given in $\mathrm{H} \varnothing \mathrm{g}$ et al. (2000, their Table 2) for the Tycho-2 accuracy was found. The only exception is the cluster Stock 2 with a width of the distribution of approx. 4 mas $\mathrm{yr}^{-1}$. We will return to this outlier in Sect. 3.2.

From the stars with a sufficiently high membership probability $(P>0.5$ to $P>0.8$, depending on the object) we derived CMDs of the clusters. We fitted isochrones based on the stellar models of Bono et al. (1997) and provided by Cassisi (private communication) to the CMDs to derive estimations for the distance moduli, reddenings, and ages of the clusters. Our initial values were those of
vL99a and Robichon et al. (1999) which in most cases led to a sufficiently good fit. For some clusters, however, the parameters had to be slightly adjusted, since the isochrones based on these results clearly did not represent the CMDs well. In those cases, which will individually be discussed in the corresponding sections, we used our own results. The metallicity was in all cases assumed to be Solar, which is, according to the Lyngå (1987) catalogue, appropriate for all clusters. The only exception may be NGC 2451 A which is metal-deficient. Values for its metallicity as given in the literature range from $Z=0.006$ to $Z=0.013$ (Strobel 1991), which, however, in terms of masses, leads to an error of a few per cent at most. The CMDs, with isochrones overplotted, are displayed in Figs. 1, 3, and 5. The parameters which are listed in Table 3 were necessary for the transformation of the $V_{T}$ magnitudes to the initial stellar masses. From $(\alpha, \delta)$ plots of the members we determined lower limits of the cluster diameters: We selected diameters outside of which almost no more members are present, assuming that these outliers are field stars with the same proper motion as the corresponding cluster. These results are added to Table 3. The "true" sizes might be larger due to mass segregation and the limited magnitude range of our study.

We found that the members of Stock 2 are differentially reddened, whereas the main sequences of the other objects either show a uniform reddening or are too sparsely populated for a distinct proposition. The variations of the reddening in the field of Stock 2 will be discussed in detail in Sect. 3.2.

We computed the masses from the $V_{T}$ magnitudes with the help of the mass-luminosity relation provided together with the isochrones. We used 6th order polynomials as an approximation. Attempts with polynomials of lower orders failed since the low masses were not sufficiently well reproduced. A comparison of our isochrones basing on the Bono et al. (1997) models with the Geneva isochrones (Schaller et al. 1992) revealed that the difference between the masses as functions of the $V$ magnitudes are less than $3 \%$. We derived the IMF slopes of the clusters 


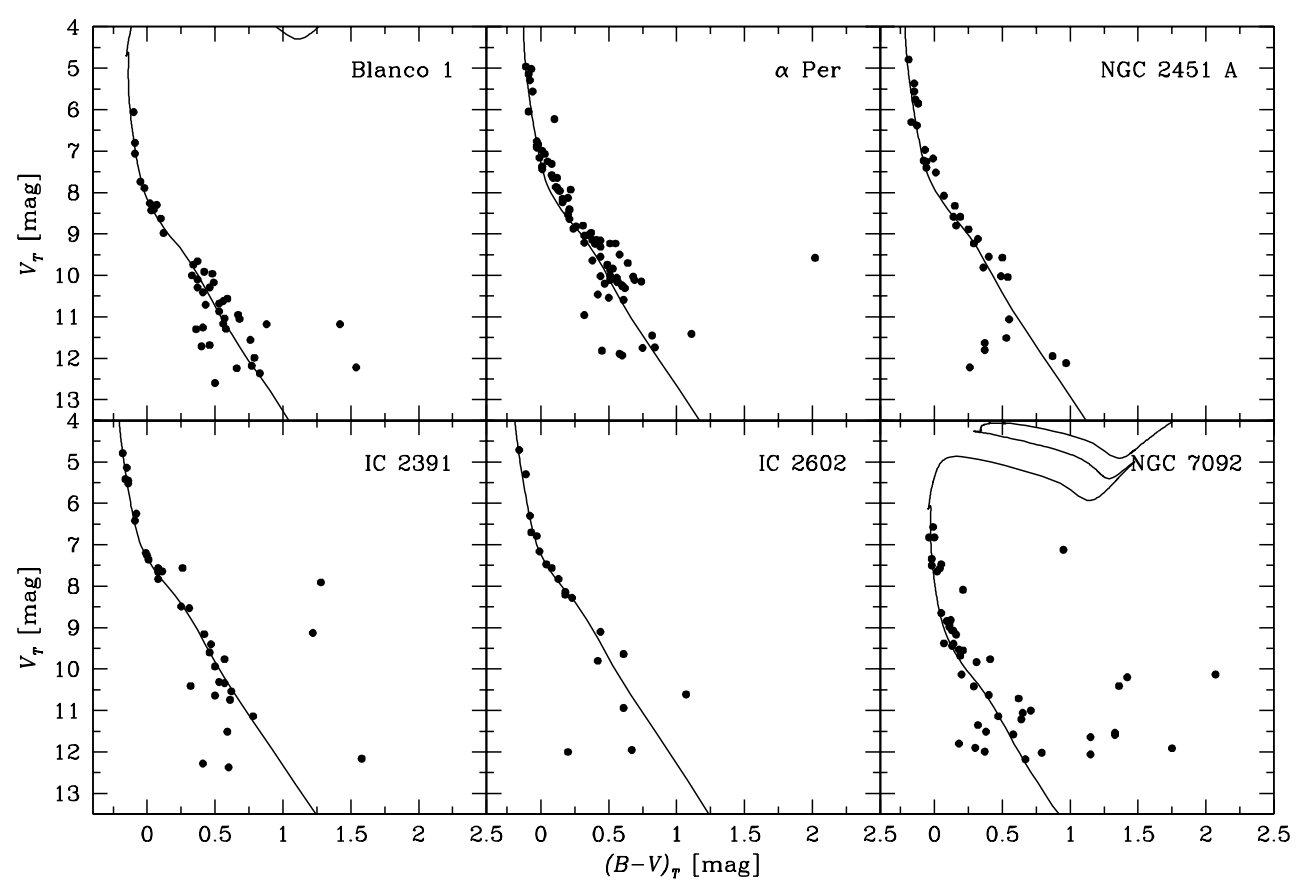

Fig. 1. CMDs of the members of six of the eight open star clusters from Tycho-2 data. We overplotted the best-fitting isochrones. The CMD of Stock 2 is discussed in more detail in Sect. 3.2, the CMD of the Pleiades is plotted in Fig. 3. Note the lack of stars fainter than $V_{T}=8 \mathrm{mag}$ in the CMD of IC 2602

Table 3. Distance modulus, reddening, and age estimations as determined during the isochrone fitting process. The determination of these parameters was necessary for the application of the mass-luminosity relation and therefore for the determination of the IMF. For a discussion of the differential reddening of Stock 2, see Sect. 3.2. Also lower limits $\underline{d}$ for the cluster diameters as derived from positional plots of the members are listed. The clusters marked with a star appear in vL99a

\begin{tabular}{|c|c|c|c|c|}
\hline cluster & $\begin{array}{c}(m-M)_{0} \\
{[\mathrm{mag}]}\end{array}$ & $\begin{array}{l}E_{B-V} \\
{[\mathrm{mag}]}\end{array}$ & $\begin{array}{c}t \\
\mathrm{Myr}\end{array}$ & $\frac{d}{\left[^{\prime}\right]}$ \\
\hline Blanco 1* & 6.8 & 0.03 & 50 & 105 \\
\hline Stock 2 & 7.5 & . & 100 & 260 \\
\hline$\alpha$ Per* & 6.3 & 0.09 & 20 & 255 \\
\hline Pleiades* & 5.6 & 0.05 & 75 & 300 \\
\hline NGC $2451 \mathrm{~A}^{*}$ & 6.4 & 0.00 & 20 & 140 \\
\hline IC $2391^{*}$ & 5.8 & 0.00 & 20 & 110 \\
\hline Praesepe* & 6.0 & 0.00 & 650 & 195 \\
\hline IC $2602^{*}$ & 5.8 & 0.03 & 10 & 185 \\
\hline NGC 7092 & 7.6 & 0.12 & 70 & 170 \\
\hline
\end{tabular}

from the masses of the members with a maximum likelihood method, i.e. we maximised the likelihood function

$\mathcal{L}(\Gamma)=\prod_{i=1}^{n} \frac{m_{i}^{\Gamma}}{\int_{m_{1}}^{m_{\mathrm{u}}} m^{\Gamma} \mathrm{d} \log m}$

with the determined masses $m_{i}$ of $n$ stars and the limits of the normalising integral being the lower and upper mass limits of the observed part of the main sequence, $m_{1}$ and $m_{\mathrm{u}}$. (For a discussion of the advantages of this method, see Sanner et al. 2000.)

A critical point of this work was the completeness of the Tycho-2 data which would have contaminated the results towards too high $\Gamma$ values, i.e. too shallow slopes. $\mathrm{H} \varnothing \mathrm{g}$ et al. (2000) state in their Table 1 that the Tycho-2 data are $99 \%$ complete down to $V_{T}=11 \mathrm{mag}$ and still $90 \%$ down to $V_{T}=11.5 \mathrm{mag}$. However, it cannot be excluded that these figures vary for different fields, depending on crowding or the first epoch material used to build Tycho-2. We tried to get an idea about the completeness of the data by the optical impression of the CMDs and by computing the IMF for different mass ranges: We assumed that the IMF indeed has the shape of a single power law within the range of the Tycho- 2 photometry. If the lower mass limit is below the completeness limit, the resulting IMF would be too shallow, since stars at the low mass end of the function would be missed. When we vary the lower mass limit up towards the completeness limit, $\Gamma$ would approach its "correct" value. Above this point, $\Gamma$ would remain on a stable level. This phenomenon is illustrated with the Stock 2 data in Fig. 2. An alternate interpretation of this behaviour of the slope as a function of the lower mass limit is that the shape of the IMF deviates from a power law with constant slope, e.g. due to a gap in the main sequence - a phenomenon which is not uncommon for open star cluster CMDs (see, e.g., the recent work of Rachford \& Canterna 2000 and references therein). Therefore, the assumption that the IMF indeed follows a "perfect" power law may lead to underestimations of the completeness limit: Read the figures given in Table 4 as upper limits (in terms of magnitudes) for the completeness limit in the fields of the clusters. Both interpretations recommend us to stop the determination of the IMF at the point where the slope becomes variable: We would either compute the IMF in a mass interval in which 


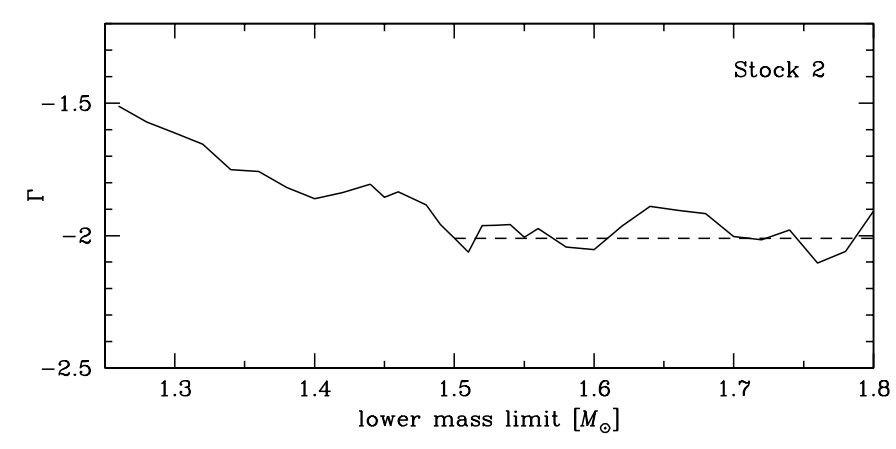

Fig. 2. IMF slope $\Gamma$ as derived for Stock 2 with our maximum likelihood technique depending on the adopted lower mass limit. The dashed line illustrates the final result of $\Gamma=-2.01$. We interpret this plot such that the data become incomplete around $m=1.5 M_{\odot}$, i.e. $V_{T}=11.4 \mathrm{mag}$. See Sect. 2 for a detailed explanation

the data are incomplete, or we would fit a single power law to an IMF which does not follow such a function.

We found varying completeness limits by this investigation. For two of the clusters, the star counts drop significantly at magnitudes as bright as $V_{T} \approx 10 \mathrm{mag}$ (see Table 4).

Most of the clusters are sparsely populated in the mass range under consideration. This made the choice of the correct completeness limit a challenging task. Figure 10 shows an overview of the IMFs of six of the clusters; the Pleiades IMF is sketched separately in Fig. 4. The results are discussed in the following section and summarised in Table 4.

An alternative way of determining the completeness limit would be the comparison of the Tycho-2 data with a second catalogue with fainter limiting magnitude, like the GSC 1.2 (Röser et al. 1998b) or USNO A2.0 (Monet et al. 1998): As long as no stars of the other catalogue are missing in Tycho-2, we can assume Tycho-2 to be complete. However, for this procedure it is required that the brightest stars missing in Tycho-2 are present in the second catalogue. This in general cannot be expected, since stars might be missing in both catalogues due to the presence of close neighbours, crowding, or similar effects. With this method we would only be able to obtain a limit at which Tycho-2 definitely is incomplete, which is not enough for a thorough IMF study.

Adding the supplement 1 of the Tycho-2 catalogue (see Høg et al. 2000 for details on the two supplements) did not affect the completeness in general: Although we found from 7 (Blanco 1 ) to 141 ( $\alpha$ Per) stars in supplement 1 , only less than 10 stars per cluster (except for $\alpha$ Per, with 13 objects) could be found for which proper motions were given. No more than four of them in each field also contained both $B_{T}$ and $V_{T}$ photometry. For the stars in Tycho-2 and its supplement 1 which are listed without proper motions, we also attempted to use the proper motions given in other Hipparcos-based catalogues, namely ACT (Urban et al. 1998) and TRC (Høg et al. 1998). For the Pleiades, this resulted in 52 additional stars. 21 of them can be considered as members according to their proper motions, but only 7 objects could be added to the IMF computation. The others either were located well below our completeness limit (4 stars), or $B_{T}$ was not given (4 stars), or the data points were so far away from the main sequence that we can assume that these data suffer from bad photometry or the stars are non-members with the same proper motions as the Pleiades (6 stars). An IMF computation with the 7 stars added led to a difference in $\Gamma$ of 0.02 compared with the IMF based on the Tycho- 2 proper motions only. This difference is so much smaller than the errors that we did not follow this procedure for the other clusters.

Comparing the proper motion centres of the clusters with the values determined by Robichon et al. (1999) using Hipparcos data, we found that the results coincide very well in both $\mu_{\alpha} \cos \delta$ and $\mu_{\delta}$ within 1 mas $\mathrm{yr}^{-1}$. This indicates that the absolute proper motions of Tycho- 2 correspond with the Hipparcos catalogue and that at least in the fields of the open clusters studied there are no systematic errors caused, e.g., by the first epoch material.

\section{Results for the individual clusters}

\subsection{Pleiades}

The first object under investigation is the Pleiades cluster. The Pleiades show a proper motion centre which is located more than 35 mas $\mathrm{yr}^{-1}$ away from the average field proper motion. In addition, previous IMF studies are available, so that we can externally check the quality of our results.

In the case of the Pleiades, the standard deviation of the member proper motions might not only reflect the inaccuracy of the Tycho-2 catalogue, but also intrinsic properties of the Pleiades, like internal velocity dispersion or perspective effects. However, since the members under consideration are located in a comparably small area (see Table 3), these effects are of minor importance.

Fitting isochrones to the CMD of the members, we found that a distance modulus of $(m-M)_{0}=5.37 \mathrm{mag}$ as given by vL99a is not the best parameter in terms of quality of the fit: The isochrone passes right in the middle of the main sequence, whereas it is our strategy to fit the isochrone towards the blue side of the main sequence to account for binary stars or Be stars, which broaden the "true" main sequence towards the red. It seems that an isochrone with $(m-M)_{0}=5.6 \mathrm{mag}$ provides a better fit. For both isochrones, a reddening of $E_{B-V}=0.05 \mathrm{mag}$ and an age of the Pleiades of $75 \mathrm{Myr}$ were assumed. The CMD with both isochrones overplotted is shown in Fig. 3. There has been a recent discussion about the reliability of the Hipparcos parallaxes on which vL99a based his distance determinations: Pinsonneault et al. (1998) as well as Narayanan \& Gould (1999) claim that small systematic errors in the Hipparcos parallaxes occur, especially in the field of the Pleiades, so that they derived a distance which corresponds to our value, while vL99a excludes these 




Fig. 3. Colour magnitude diagram of the Pleiades members. We overplotted the Bono et al. (1997) isochrones for $(m-M)_{0}=5.37 \mathrm{mag}$ (dotted line) and $(m-M)_{0}=5.6 \mathrm{mag}$ (dashed line). Both isochrones are reddened by $E_{B-V}=$ 0.05 mag. The star marked with a cross is listed as a blue straggler candidate in Ahumada \& Lapasset (1995). This object is discussed in the text (Sect. 3.1)

errors as a consequence of extensive tests of the Hipparcos data (see also van Leeuwen 1999b).

We cannot resolve this problem with our results, therefore we computed the IMF based on both parameter sets leading to results of $\Gamma=-1.99 \pm 0.39$ and $\Gamma=$ $-2.10 \pm 0.39$, with our and vL99a's distances, respectively. This means that within the errors the IMF determination does not depend on the uncertainties of the distance determination. The two mass functions are sketched in Fig. 4. In Tables 3 and 4 we retain our values.

In a study of the IMF of the Pleiades, almost in the same mass range as ours, van Leeuwen (1980) arrived at a result of (transformed to our notation) $\Gamma=-1.71 \pm 0.27$ based on the members known at that time. Within the errors, this is in good agreement with our values. Meusinger et al. (1996) studied the IMF down to approx. $0.4 M_{\odot}$ and stated that globally the Pleiades IMF cannot be represented by a single power law. Therefore they did not provide a specific slope for $m \gtrsim M_{\odot}$, but they noted their IMF as being "somewhat steeper" than van Leeuwen's (1980) result in the corresponding mass interval, so that their findings seem to support our result very well.

A region with a radius of $7^{\circ}$ around the centre of the Pleiades contains 397 stars which are common to both the Hipparcos and Tycho-2 catalogues. This gave us a good opportunity to compare the proper motions of the two catalogues. We found a good agreement with $\Delta \mu_{\alpha} \cos \delta=$

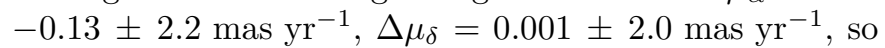

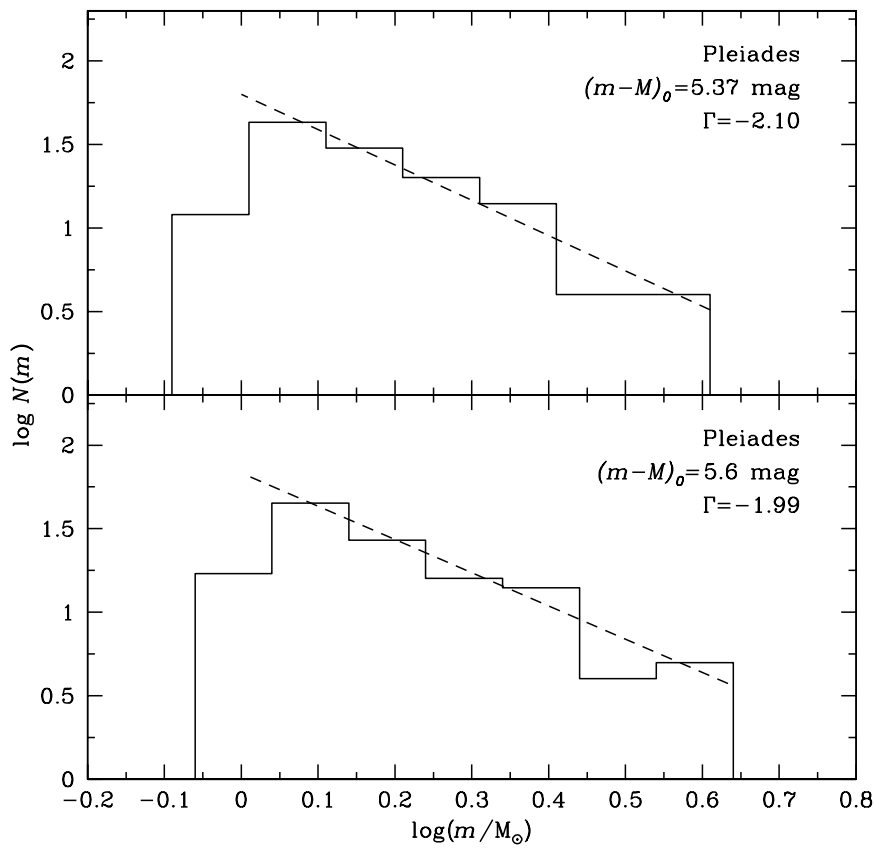

Fig. 4. Initial mass functions of the Pleiades cluster. The upper diagram is based on a distance modulus of $(m-M)_{0}=$ $5.37 \mathrm{mag}$, the lower one on $(m-M)_{0}=5.6 \mathrm{mag}$. The dashed lines symbolise the power laws with slopes of $\Gamma=-2.10$ and $\Gamma=-1.99$, respectively, the limits of the lines the mass interval under consideration. Note that, although we plot an underlying histogram for illustration, the functions were determined with a maximum likelihood method. The rightmost bins contain only a few stars, so that the bins in this range are not meaningful

that the absolute reference systems of both catalogues appear to be almost identical.

One of the three stars mentioned in the list of Ahumada \& Lapasset (1995) as blue stragglers of the Pleiades can be found in the Tycho-2 main catalogue; the other two are too bright, so that no proper motions were determined for them. The object included in Tycho-2, HD 23338, has a proper motion of $\mu_{\alpha} \cos \delta=$ $+20.1 \mathrm{mas} \mathrm{yr}^{-1}, \mu_{\delta}=-44.8$ mas $\mathrm{yr}^{-1}$, corresponding to a membership probability of $P=0.98$, so that there is clear evidence that this object is a member of the Pleiades. We marked this star in the CMD in Fig. 3 with a cross. The star is located to the left of the best fitting isochrone, however it is still well below the turn-off point of the main sequence so that we do not see evidence for the blue straggler nature of this object. For the other two objects, we can refer to the Hipparcos proper motions instead: As stated before, the proper motions of Hipparcos and Tycho-2 are almost identical, so that the pure Hipparcos figures can be taken as an indication about membership without any transformation or other special treatment, although we do not give explicit membership probabilities. In the case of the Pleiades, the two stars not in Tycho-2 are HD 23302 (HIP 17499) and HD 23630 (HIP 17702). Their proper motions are close to the centre of proper motions of the Pleiades so that we can assume that these objects indeed 

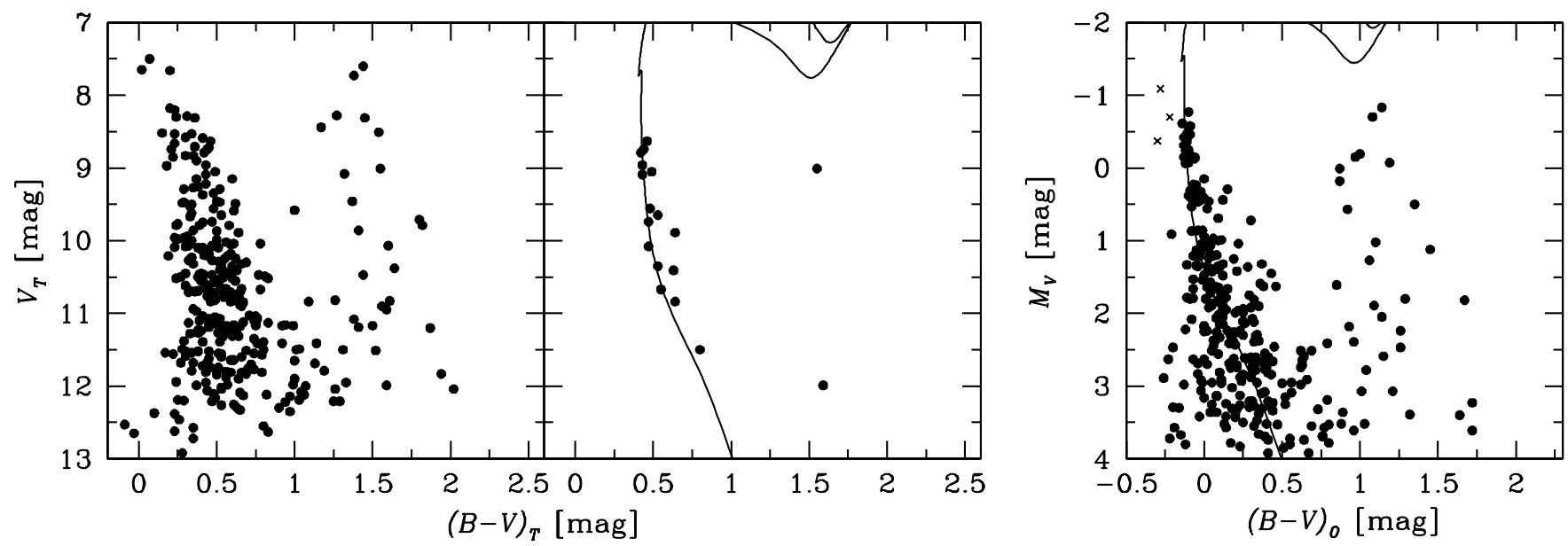

Fig. 5. Colour magnitude diagrams of the members of Stock 2. The leftmost diagram shows all members with their apparent magnitudes. Note the broad main sequence which inspired us to study the differential reddening of the cluster. The middle diagram shows the subfield of Stock 2 which is accentuated in Fig. 6 . This field provided the evidence for the distance modulus of Stock 2, determined as $(m-M)_{0}=7.5$ mag. Overplotted is the isochrone which best fit these data, the reddening is $E_{B-V}=0.55 \mathrm{mag}$, the adopted age $t=100 \mathrm{Myr}$. The right panel is the de-reddened CMD of Stock 2 with absolute magnitudes. We overplotted the $100 \mathrm{Myr}$ isochrone. The red $\left((B-V)_{T} \gtrsim 1 \mathrm{mag}\right)$ stars are most likely mis-identified field stars which by chance have the same proper motion as the cluster. The blue stars marked with crosses are either caused by the same factor or have a smaller reddening than the the region they are assigned to in our rough reddening map (Fig. 6)

belong to the Pleiades. Note that since these two objects do not appear in the Tycho-2 catalogue with complete sets of data, we did not include them in our membership analysis and therefore they are not present in the CMD of the Pleiades members (Fig. 3).

Slettebak (1985) provides data on Be and shell stars in open star clusters. He reports four members of the Pleiades. The proper motions of two of these stars, HD 23302 (17 Tau) and HD 23630 ( $\eta$ Tau) are not listed in Tycho-2, but are in the Hipparcos catalogue. Both objects are likely members of the Pleiades according to their Hipparcos proper motions. The two remaining stars of the Slettebak (1985) list, HD 23480 (23 Tau) and HD 23862 (28 Tau) are cluster members with membership probabilities of $P=0.97$, so that Tycho-2 supports the proposition that these objects are members of the Pleiades, too. It is interesting that HD 23302 appears in both lists. However, it should be remarked that its category in Ahumada \& Lapasset (1995) is 3, i.e. only weak evidence for a blue straggler nature, whereas Slettebak's (1985) discussion seems to be more sure.

\subsection{Stock 2}

Stock 2 is by far the most populous cluster (down to the limiting magnitude of Tycho-2) of our sample. There has been a controversial discussion about the distance modulus and reddening of the cluster (see the overview in the recently published work of Foster et al. 2000). Here we just summarise that distance moduli from $7.4 \mathrm{mag}$ (Piskunov 1980) up to $8.36 \mathrm{mag}$ (Pandey et al. 1989) are proposed; the reddenings found are of the order of $E_{B-V} \approx 0.3 \mathrm{mag}$.

Our CMD of the members (Fig. 5, left diagram) shows a broad $(\approx 0.3 \mathrm{mag})$ main sequence over the entire magnitude interval, so that we decided to study the CMDs of smaller areas to find out whether Stock 2 is differentially reddened. We found that indeed for small portions of the data the main sequences are much narrower, and we found different reddening values: Large portions of the field of Stock 2 are reddened by $E_{B-V}=0.3 \mathrm{mag}$, however, we found areas with reddenings from $E_{B-V}=0.1 \mathrm{mag}$ up to $0.55 \mathrm{mag}$. The results of this investigation are presented in the reddening map of Stock 2 (Fig. 6). Since the single fields do not cover many objects, due to the bright limiting magnitude of Tycho-2, a much more detailed reddening map could be achieved with a deeper photometric study of Stock 2.

While for most areas isochrones with a large range of distance moduli $\left(7.5 \mathrm{mag} \lesssim(m-M)_{0} \lesssim 8.2 \mathrm{mag}\right)$ led to good fits, one field $\left(2^{\mathrm{h}} 14^{\mathrm{m}} 24^{\mathrm{s}} \leq \alpha_{2000} \leq 2^{\mathrm{h}} 15^{\mathrm{m}} 0^{\mathrm{s}}\right.$, $59^{\circ} 18^{\prime} \leq \delta_{2000} \leq 59^{\circ} 39^{\prime}$, marked in Fig. 6) could not be sufficiently well fitted after applying a distance modulus of more than $(m-M)_{0}=7.5 \mathrm{mag}$. The CMD of this field together with the corresponding isochrone is sketched in Fig. 5, middle diagram. On the other hand, all the other fields do correspond to a distance modulus of $(m-M)_{0}=7.5 \mathrm{mag}$. Therefore we adopt this value to represent the distance modulus of Stock 2 . The right diagram of Fig. 5 shows the composition of the de-reddened CMDs of the individual sub-fields on the basis of absolute magnitudes. It is evident that the main sequence is much more pronounced than the original CMD (left diagram 


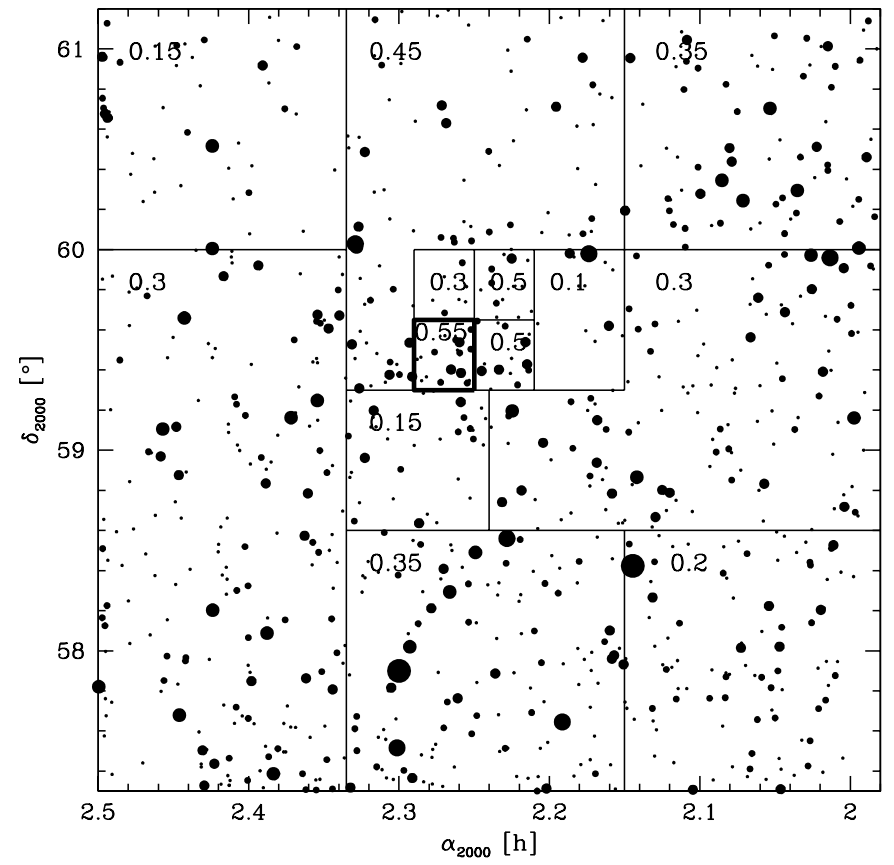

Fig. 6. Reddening map of Stock 2. The largest dots represent $V_{T}<6$ mag stars, the smallest ones are still brighter than $V_{T}=11$ mag. North is up, east to the left. The fields could not be chosen arbitrarily small in size, as it had to be taken into account that the fields cover a sufficient amount of stars to see the relevant features of the main sequence. The CMD of the field marked with heavy borders and a reddening of $E_{B-V}=0.55 \mathrm{mag}$ is plotted in Fig. 5, middle diagram. This field turned out to be important for the distance determination of Stock 2 (see Sect. 3.2)

in Fig. 5), at least down to $M_{V}=2 \mathrm{mag}$. With a more detailed reddening map the CMD could still be improved.

The CMD shows three bright very blue $\left((B-V)_{T}<\right.$ $-0.2 \mathrm{mag}$, marked in the right diagram of Fig. 5) as well as numerous red $\left((B-V)_{T}>1 \mathrm{mag}\right)$ stars that do not fit to the selected isochrone. These objects can be explained as randomly having the same proper motions as Stock 2, but in fact being field stars. The objects are uniformly distributed over the field in an $(\alpha, \delta)$ plot; for example, two of them can be found in the CMD of the selected region of Stock 2 mentioned before (Fig. 5, middle diagram). Another explanation for the blue objects would be that they are less reddened than proposed for the field in which they are located.

The IMF was computed from 262 main sequence stars within a field of view of $4^{\circ} \times 4^{\circ}$, after some 70 too red and blue stars discussed before had been removed. 204 objects are located above the completeness limit of $V_{T}=$ $11.0 \mathrm{mag}$, so that the slope of $\Gamma=-2.01 \pm 0.40$ finally is based on these stars.

We had mentioned in Sect. 2 that the standard deviation of the members of Stock 2 was larger than for all the other clusters and higher than expected from the general accuracy of the Tycho-2 catalogue. Stock 2 is located within the Vatican declination zone $\left(+55^{\circ}\right.$ to $\left.+64^{\circ}\right)$ of the Astrographic Catalogue. A remark of Eichhorn (1974, p. 297) that the accuracy of the Vatican measurements are comparably low could explain this substandard behaviour.

Five stars in the field of Stock 2 are mentioned by Ahumada \& Lapasset (1995) as blue stragglers belonging to the cluster. Three of them - their stars 6, 31 (HD 13402), and 45 - show membership probabilities of $P=0$. The other two - stars 110 (HD 13735) and 138 (HD 13909) - have high probabilities of $P \approx 0.97$, however, after the de-reddening process, they are located well in the main sequence of the CMD. Therefore, we propose that all five objects are not blue straggler members of Stock 2 .

\subsection{Praesepe (NGC 2632, M 44)}

Astrometric and photometric aspects of Praesepe have been well studied before (e.g. Jones \& Cudworth 1983; Jones \& Stauffer 1991), including IMF studies (Williams et al. 1995; Pinfield et al. 1999).

The Tycho-2 stars which were determined as members of Praesepe mostly are located within a distance of $1.5^{\circ}$ of the centre of the cluster. The CMD shows a scattered main sequence, so that a differential reddening seems likely. Since the total number of detected member stars brighter than $V_{T}=10 \mathrm{mag}$ is only 79 , a meaningful reddening map as determined for Stock 2 (Sect. 3.2) could not be obtained. The isochrone shifted according to the vL99a results fits the main sequence quite well, however, the isochrone lies slightly too red, so that we propose a reddening of $E_{B-V}=0.02 \mathrm{mag}$.

Due to the age of Praesepe, which we determined to be $650 \mathrm{Myr}$, only a small range of main sequence stars could be covered. Therefore, it was not possible to derive a trustworthy IMF from the Tycho-2 data.

Ahumada \& Lapasset's (1995) catalogue of blue stragglers contains five stars in the region of Praesepe. Three of them, HD 73618, HD 73666 (40 Cnc), and HD 73819 have proper motions leading to high membership probabilities $(P>0.9)$. HD 73711 shows an intermediate probability of $P=0.79$, but it is still likely that this object belongs to Praesepe. The final object, HD 73120, is a clear nonmember with $P=0$.

\section{4. $\alpha$ Per (Melotte 20)}

Although numerous photometric and kinematic studies are available for the $\alpha$ Per cluster (e.g. Mitchell 1960; Fresneau 1980; Prosser 1992, 1994), the IMF of this object has not been investigated since the work of Tarrab (1982).

The vector point plot diagram of the $\alpha$ Per cluster is presented in Fig. 7. The good separation between field and cluster proper motions is clearly visible: Only very few field stars might erroneously be identified as cluster members. The width of the distribution of the cluster stars is $\sigma \approx 1.5$ mas $\mathrm{yr}^{-1}$ which coincides with the proposed standard deviation for individual objects included in the 


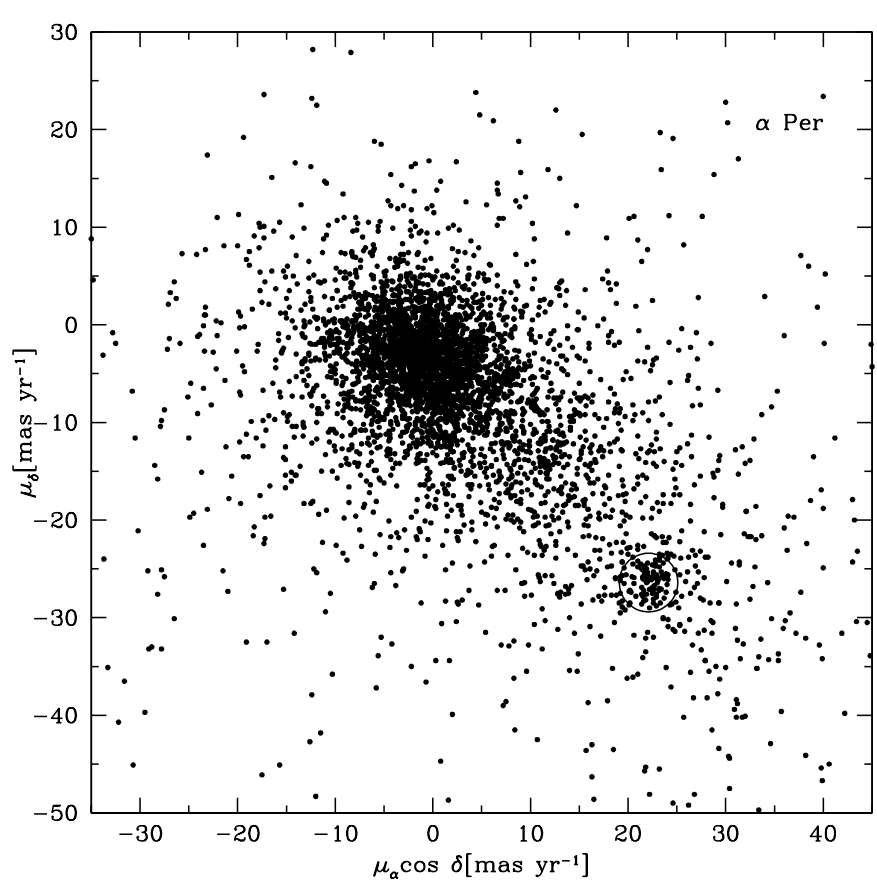

Fig. 7. Vector point plot diagram of the stars within $5^{\circ}$ of the centre of the $\alpha$ Per cluster. The cluster is clearly visible in the lower right part of the diagram. A circle is plotted around the region inside which the stars are considered members. This figure contains 4460 objects, 81 of which are determined to belong to the $\alpha$ Per cluster

Tycho-2 catalogue. A circle indicates which stars were selected as the members of the $\alpha$ Per cluster.

vL99a found values of $(m-M)_{0}=6.31 \mathrm{mag}$ and $E_{B-V}=0.09 \mathrm{mag}$ for the $\alpha$ Per cluster. We could confirm these values with the Tycho-2 data. On the other hand, together with the proposed age of $50 \mathrm{Myr}$, the upper part of the main sequence is not well represented by the fitted isochrone. We prefer a younger isochrone with $t=20 \mathrm{Myr}$, which is plotted in Fig. 1. A higher age could only be explained by the brightest members detected being blue stragglers.

Three stars are listed as blue stragglers by Ahumada \& Lapasset (1995). Two of them, HD 22928 and HD 24760, are too bright to be included in the Tycho- 2 catalogue with proper motions. One of them, HD 21428, has a membership probability of $P=0.34$ according to its Tycho-2 proper motion, so that it can be assumed that this object is a field star. The proper motions of the other two, HD 22928 (HIP 17358) and HD 24760 (HIP 18532), can again be taken from the Hipparcos catalogue: Both have a proper motion which indicates they are not members of $\alpha$ Per, since for HD 22928 the deviation of $\mu_{\delta}$ from the

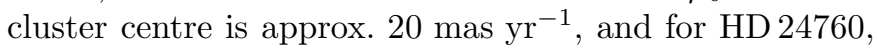
$\mu_{\alpha} \cos \delta$ is too far away from the value for the members.

In Slettebak (1985), three objects are listed as Be or shell stars in the region of the $\alpha$ Per cluster. HD 21551 has a membership probability of $P=0.39$ and HD $22192(\psi$ Per) of $P=0.27$ which means that both objects are likely non-members. HD 25940 (48 Per) is not included in the
Tycho-2 catalogue, but it is a Hipparcos star (HIP 19343)

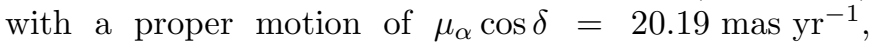
$\mu_{\delta}=-33.26$ mas $\mathrm{yr}^{-1}$. Comparing this with the proper motion centre of the cluster (Table 1), this means that this star, too, shows a proper motion which does not coincide with the motion of the cluster. Only the fourth star, HD 21455, mentioned in the text of Slettebak (1985) as a non-member, has a membership probability of $P=0.76$ which makes it a likely cluster member from the proper motion point of view, since the probability at which we distinguished members and non-members in the case of $\alpha$ Per was $P=0.6$. Since Slettebak (1985) mentions rotational velocities from Kraft (1967) and radial velocities from Petrie \& Heard (1969) as further membership criteria, it is still possible that this object in fact is not a member of the cluster.

\subsection{Blanco 1}

Blanco 1 (also referred to as the $\zeta$ Scl cluster) turned out to be the cluster with the second faintest completeness limit of our sample with $V_{T}=11.4 \mathrm{mag}$, following the rough method presented earlier in Sect. 2. Nevertheless, the IMF was computed on the basis of 34 stars only. The distance moduli of Blanco 1 according to vL99a $6.82 \mathrm{mag}$ - and Robichon et al. (1999) - $7.1 \mathrm{mag}$ - differ by almost $0.3 \mathrm{mag}$. We found that an isochrone on the basis of the vL99a value fits the CMD of the members slightly better, but the Robichon et al. (1999) distance modulus cannot be excluded, either. Instead of a reddening of $E_{B-V}=0.09 \mathrm{mag}$, as proposed by vL99a, we suggest a lower value of $E_{B-V}=0.03 \mathrm{mag}$. This value is in good agreement with other studies of Blanco 1 , e.g. Westerlund et al. (1988). The cluster age as given in vL99a could be well reproduced. The luminosity function of Blanco 1 (see Fig. 8) shows a gap in the range from approx. $V_{T}=9 \mathrm{mag}$ to $V_{T}=9.7 \mathrm{mag}$ (corresponding to masses from $m=1.6 M_{\odot}$ to $\left.m=1.9 M_{\odot}\right)$. When experimenting with the lower mass limit during the IMF determination, this led to a temporary increase of $\Gamma$ from -2.27 to as much as -1.12 , which normalised after passing the gap. It must be remarked that only 11 members have masses higher than $1.9 M_{\odot}$, so that the IMF can most reliably be described with the help of the lower mass stars. The gap seems not to be a consequence of incomplete Tycho-2 data, since it has been observed before (Eggen 1983). In addition, these gaps are not uncommon for open clusters (Rachford \& Canterna 2000). As a consequence, Blanco 1 is an example of a star cluster for which a power law does not well describe the shape of the entire IMF. This is also illustrated by the comparably high error of the maximum likelihood fit. It would be interesting to obtain a deeper photometric study of this object to find out whether the IMF shows a "normal" power law shaped behaviour for lower mass stars or if further gaps appear. An IMF computed on the basis of the vL99a distance modulus leads to a slope of $\Gamma=-2.27 \pm 0.70$. 


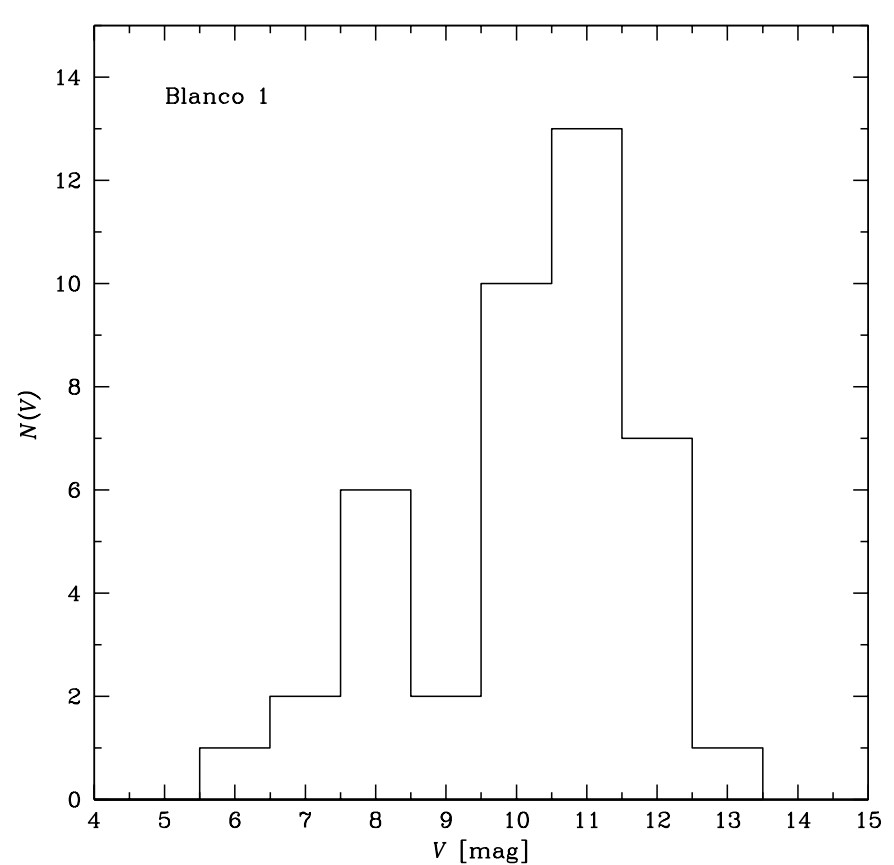

Fig. 8. $V_{T}$ luminosity function of Blanco 1. Note the dip around $V_{T}=9 \mathrm{mag}$

The star $\zeta$ Scl itself is, according to Ahumada \& Lapasset (1995), a blue straggler cluster member. For this object we find a membership probability of $P=0$ as a consequence of its proper motion of $\mu_{\alpha} \cos \delta=$

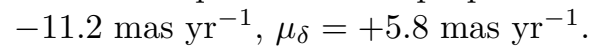

\subsection{NGC 2451 A}

NGC 2451 has been the subject of a discussion regarding whether it really is an open star cluster or not (Eggen 1983, 1986; Röser \& Bastian 1994). Recently, Carrier et al. (1999) found evidence that this object actually consists of two clusters in the same line of sight, as proposed earlier by Röser \& Bastian (1994). Baumgardt (1998) was only able to confirm existence of one of the two clusters on the basis of the Hipparcos and ACT catalogues. Carrier et al. (1999) named the two objects NGC 2451 A and B. We will stick to this nomenclature in the following. Cluster A, which is the target of our study, is located at a distance of approx. $190 \mathrm{pc}$, whereas component B is $360 \mathrm{pc}((m-$ $M)_{0}=7.8 \mathrm{mag}$ ) away from the Sun. NGC $2451 \mathrm{~B}$ will not be studied here, as its proper motion is too close to the centre of the field proper motion.

For NGC $2451 \mathrm{~A}$, we found that adopting the vL99a reddening of $E_{B-V}=0.04 \mathrm{mag}$ leads to an isochrone which is too far redwards to properly represent the main sequence. vL99a does not distinguish between components $\mathrm{A}$ and B. However, the values given for the proper motions (his Table 1) indicate that he - as well as Robichon et al. (1999) - really does investigate component A. Carrier et al. (1999) found the reddening to be $E_{B-V}=0.01 \mathrm{mag}$, which better corresponds to our isochrone fit. Due to the lack of evolved stars, the age determination is a critical task: A 36 Myr isochrone fits the data as well as a $10 \mathrm{Myr}$ isochrone. Carrier et al. (1999) seem to overestimate the age of NGC $2451 \mathrm{~A}$ with their value of $50 \mathrm{Myr}$.

With $\Gamma=-0.69$, NGC 2451 A shows the shallowest IMF of our sample. Since the usable portion of the main sequence consists of 27 stars only, this result is quite uncertain. Still, we can conclude that the IMF of this object is not as steep as Scalo's (1998) average slope of $\Gamma=-1.7$ would suggest.

Ahumada \& Lapasset (1995) mention two stars in the field of NGC 2451 as blue stragglers. One of them, HD 61831, is a likely cluster member with a probability of $P=0.9$. However, since it is located slightly right of the fitted isochrone in the $\mathrm{CMD}$, its nature as a blue straggler is doubtful. On the other hand, the second object, HD 63465, has a proper motion of $\mu_{\alpha} \cos \delta=$

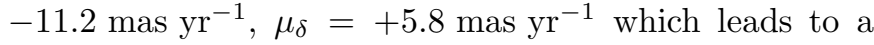
membership probability of $P=0$. Following Carrier et al. (1999) who indirectly give evidence for the proper motion of cluster B with the statement that HR 2968

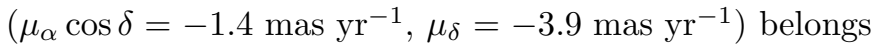
to NGC 2451 B, HD 63465 does not seem to be a member of either of the clusters.

\subsection{IC 2391}

As for NGC 2451 A, IC 2391 seems to be less reddened than proposed by vL99a. Our result that IC 2391 is unreddened can also be found, e.g., in Perry \& Hill (1969). The main sequence of IC 2391 (see Fig. 1) is not very evenly populated: Several gaps of $\Delta V_{T} \approx 0.7 \mathrm{mag}$ can be found around $V_{T}=6 \mathrm{mag}, V_{T}=7 \mathrm{mag}$, and $V_{T}=8.25 \mathrm{mag}$. Below $V_{T}=11 \mathrm{mag}$ only very few stars with the proper motion of the cluster are present in the CMD, and they are widely spread in colour. It seems that the data are complete down to $V_{T} \approx 11 \mathrm{mag}$. IC 2391 in general is sparsely populated, so that we had to compute its IMF on the basis of only 29 stars. The result, $\Gamma=-1.07 \pm 0.53$, again is at the shallow end of the IMF slope interval.

\subsection{IC 2602}

IC 2602 shows a clear and narrow main sequence down to $V_{T}=8 \mathrm{mag}$. Below this point, almost no stars are found, and the few detected members are widely scattered in the CMD. In a positional plot of all stars with $8 \mathrm{mag} \leq V_{T} \leq 11.5 \mathrm{mag}$ of the Tycho-2 data (see Fig. 9) one finds that in a $1^{\circ}$ wide area around the centre of IC 2602, almost no stars are found. An equivalent plot of, e.g., the GSC, Version 1.2, (Röser et al. 1998b) does not show this decrease in stellar density. This might mean that for some reason the stars fainter than $V_{T}=8 \mathrm{mag}$ in this area - and hence members of the cluster - are not included in the Tycho-2 catalogue. The total stellar density, as one can see in images of this region, is low enough so that neither crowding nor neighbouring bright stars might have disturbed the measurements of either the Tycho star 


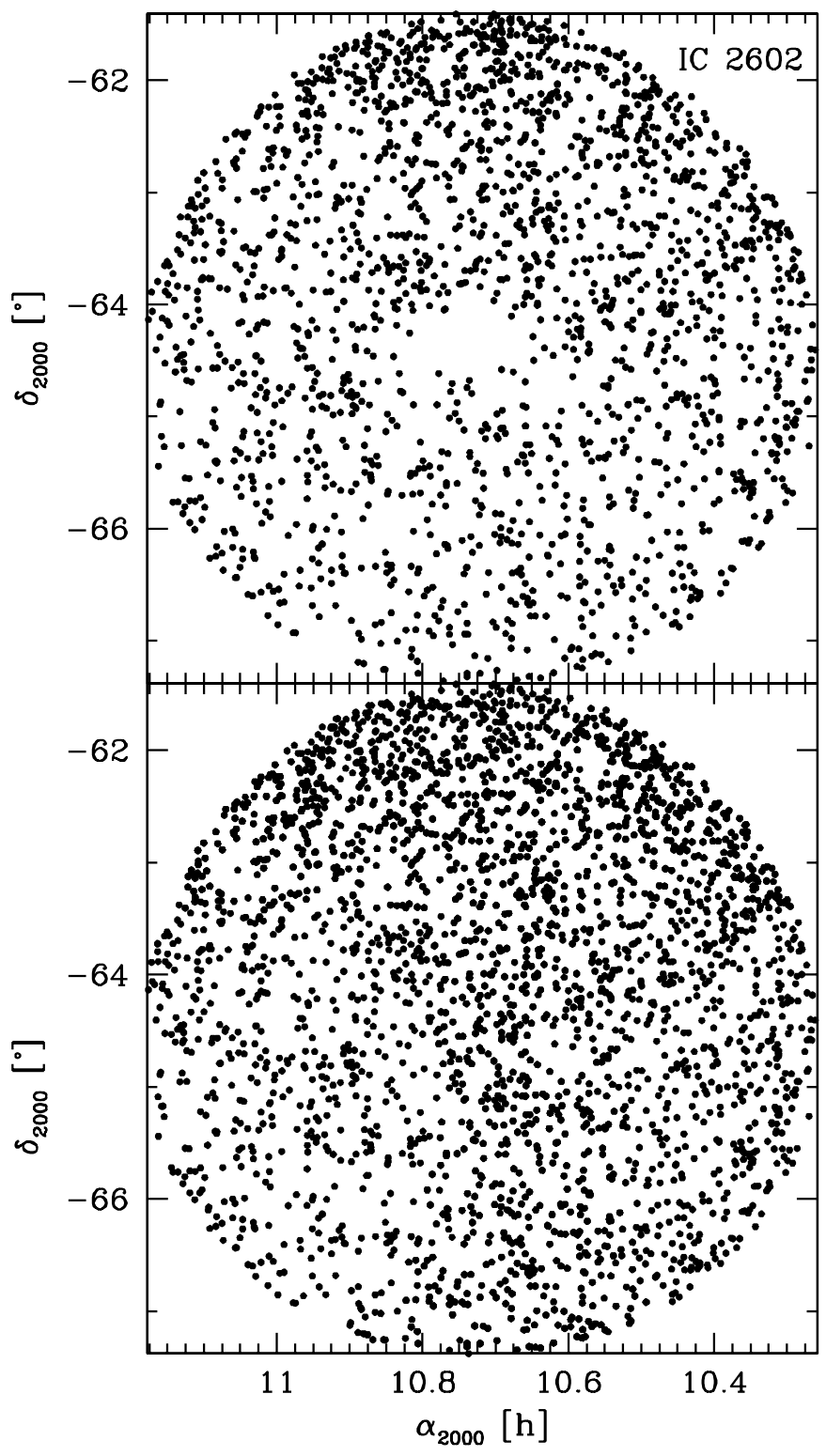

Fig. 9. Plots of the stars in the region of IC 2602 in the magnitude range $8 \mathrm{mag} \leq V_{T} \leq 11.5 \mathrm{mag}$ of the Tycho-2 stars (upper diagram) and GSC 1.2 (lower diagram). The radius of the field of view is $3^{\circ}$. Note the lack of stars in this magnitude range in the Tycho- 2 data

mapper or the first epoch data. We also checked the stars in Tycho-2 that are listed without proper motions and the two supplements of Tycho-2. Supplement 1 contains 31 stars, but only for one of them is a proper motion given. There is an above-average number of these stars in the region of IC 2602, but when we added all these objects, the total stellar numbers are still too low. The original Tycho catalogue does not show this lack of stars.

$V_{T}=8 \mathrm{mag}$ corresponds to a mass of $m=1.9 M_{\odot}$, and the number of members which could be used for the IMF computation was no more than ten, too small a number to compute the mass function of IC 2602.

Ahumada \& Lapasset (1995) list a "well known blue straggler" for IC 2602: $\vartheta$ Car. It indeed has a Hipparcos
Table 4. IMF slopes $\Gamma$ of the star clusters under consideration together with the formal errors derived during the maximum likelihood analysis. Also the mass and $V_{T}$ magnitude ranges which could be taken into account are listed. The upper limit of the $V_{T}$ interval can be taken for an estimation of the completeness limit of the Tycho-2 data in the regions of the clusters. For this point, see the remarks given in Sect. 2. The numbers of stars given are valid for the members within these intervals

\begin{tabular}{lrccc}
\hline cluster & $\#$ & $\Gamma$ & $\begin{array}{c}\text { mass range } \\
{\left[M_{\odot}\right]}\end{array}$ & $\begin{array}{c}V_{T} \text { range } \\
{[\mathrm{mag}]}\end{array}$ \\
\hline Blanco 1 & 34 & $-2.27 \pm 0.70$ & {$[1.1 ; 4.8]$} & {$[6.1 ; 11.4]$} \\
Stock 2 & 204 & $-2.01 \pm 0.40$ & {$[1.5 ; 4.1]$} & {$[7.6 ; 11.0]$} \\
$\alpha$ Per & 70 & $-1.57 \pm 0.44$ & {$[1.1 ; 6.8]$} & {$[5.0 ; 10.5]$} \\
Pleiades & 127 & $-1.99 \pm 0.39$ & {$[1.0 ; 4.1]$} & {$[5.0 ; 10.9]$} \\
NGC 2451 A & 27 & $-0.69 \pm 0.63$ & {$[1.3 ; 6.8]$} & {$[4.8 ; 10.0]$} \\
IC 2391 & 29 & $-1.07 \pm 0.53$ & {$[1.1 ; 8.1]$} & {$[3.5 ; 10.7]$} \\
NGC 7092 & 25 & $-1.93 \pm 1.24$ & {$[1.4 ; 3.4]$} & {$[6.5 ; 9.9]$} \\
\hline
\end{tabular}

proper motion close to the centre of the cluster proper motion.

\subsection{NGC 7092 (M 39)}

For the sparse open cluster NGC 7092, reddenings of a few hundredths of a magnitude can be found in the literature (e.g., Mohan \& Sagar 1985; Manteiga et al. 1991; Nicolet 1981), however, we found that no stars in the entire field studied (members and non-members) justify an isochrone reddened by less than $E_{B-V}=0.1 \mathrm{mag}$. Concerning the distance modulus, our result of $(m-M)_{0}=7.6 \mathrm{mag}$ is in good agreement with other studies. The determination of the IMF slope of NGC 7092 led to a high error of $\Delta \Gamma>1$. This cannot only be explained with the low number of 25 members above the estimated completeness level, which, with $V_{T}=9.9 \mathrm{mag}$, is the brightest of the entire study besides IC 2602. Another point is the shape of the IMF which cannot be well represented by a power law: The CMD of NGC 7092 shows a magnitude region with (almost) no stars, in this case for $7.7 \mathrm{mag} \lesssim V_{T} \lesssim 8.6 \mathrm{mag}$. In terms of masses, this interval corresponds to $1.8 M_{\odot} \lesssim m \lesssim 2.4 M_{\odot}$. This again underlines the advantages of a maximum likelihood estimation compared with a least square fit to an IMF histogram, which would have led to an error of $\Delta \Gamma= \pm 0.14$ only, so that the unusual shape of the IMF would not have been striking.

\section{Summary and discussion}

We used the information of the Tycho-2 catalogue for the determination of the IMF of nearby open star clusters in the $\gtrsim 1 M_{\odot}$ mass range and to demonstrate the capabilities of Tycho-2.

The IMF of seven open star clusters could be represented by power laws with exponents ranging from $\Gamma=$ -0.69 to $\Gamma=-2.27$. Within the errors, this is in good agreement with the range of IMF slopes as given, e.g., by Scalo (1998). An average slope from our results could 


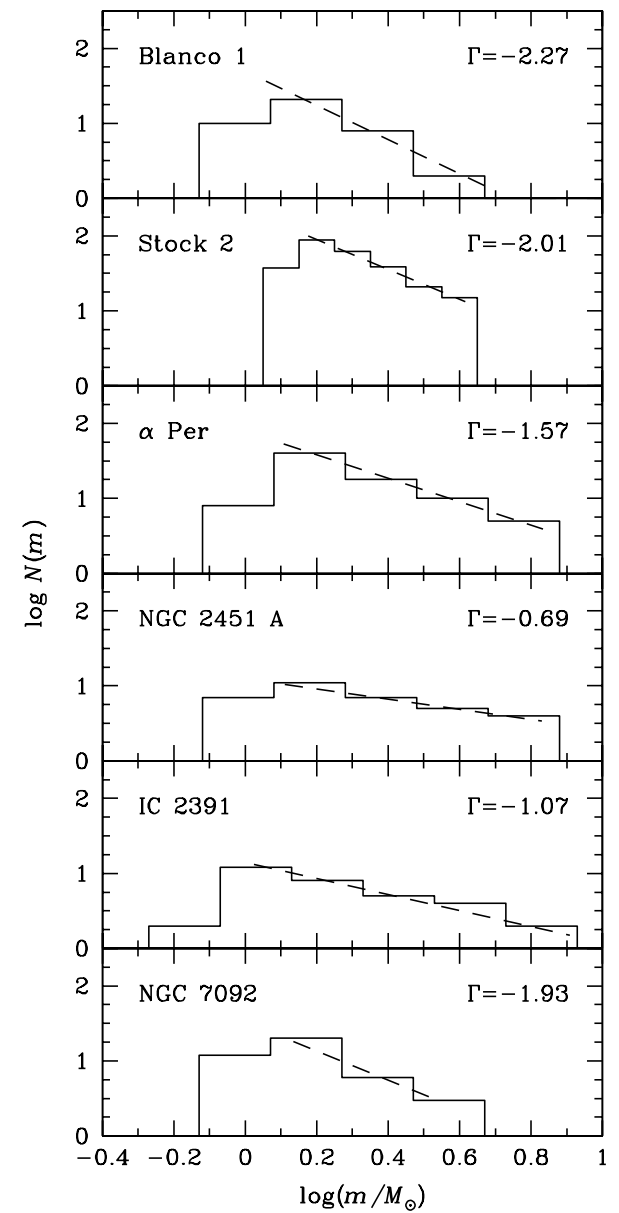

Fig. 10. Initial mass functions of six of the open star clusters using Tycho-2 data. The Pleiades IMF can be found in Fig. 4. For Praesepe and IC 2602, no reliable IMF could be determined and their histograms are not shown. For more details about the plots, see the explanations in Fig. 4

be computed as $\Gamma=-1.65$. The standard deviation of the sample of slopes equals $\Delta \Gamma=0.6$, whereas the standard deviation as derived from the individual errors of the slopes is only $\Delta \Gamma=0.26$.

Two of the clusters, Blanco 1 and NGC 7092, show significant gaps over almost $1 \mathrm{mag}$ in luminosity or a few tenths of a Solar mass, influencing the slope determined and their errors. For these clusters, further studies with fainter limiting magnitudes will be needed to find a more precise IMF, since not only would the mass range be larger, but also the numbers of members to be detected.

With a Monte Carlo simulation, we tested whether our assumption that the IMF of the clusters can be represented with a (single) power law within the mass range covered by our study: We repeatedly simulated the mass functions of a cluster with the same number of members as found from Tycho-2 and with the slope derived from the data. From these samples, we determined the slopes with the same method as for the actual objects. A probable difference between the errors as found from the maximum likelihood method and the standard deviation of the $\Gamma$ values found from the simulation might be an indication that the IMF of that object does not follow a power law. All clusters show an error smaller by 0.15 in the simulated clusters. This could not only be explained by the fact that the "true" IMF of the clusters does not follow a power law, but also by the slight contamination of the data with field stars (See the remarks in Sect. 2). The influence of these objects at arbitrary distances cannot be well simulated in the Monte Carlo experiments. The only exception is NGC 7092, with a difference of 0.4. The gaps in the main sequence mentioned in Sect. 3.9 and the low number of total stars might be the cause for this behaviour. However, we do not find evidence in any of the clusters that the choice of a power law as the representative of the IMF was clearly incorrect.

Comparing our results with the study of Tarrab (1982), we find good agreement for four of the five clusters which are discussed in both publications. Only the slope of NGC 7092, for which Tarrab found - in our notation $-\Gamma=-0.72$, dramatically differs from our value of $\Gamma=-1.93$.

Tycho-2 allows us to discuss the membership of individual objects mentioned previously in the literature. We inspected the catalogue of blue stragglers of Ahumada \& Lapasset (1995). Many of their objects belong to their "class 3", which means that there is only weak evidence (like the location in a CMD) for stars being blue stragglers and members of the clusters. For these objects, if they are bright enough, proper motions are now available, so that the list can be improved by cleaning it of field stars. The second paper under consideration was Slettebak's (1985) list of Be and shell stars in open clusters. While we could confirm the list for the Pleiades, the nature of the stars mentioned in the context of the $\alpha$ Per cluster has to be revised.

Our analysis showed that the completeness given for the Tycho-2 catalogue by Høg et al. (2000) is too optimistic, at least in several fields of the clusters studied: A field with a diameter of approx. $1^{\circ}$ around the centre of IC 2602 contains just a few stars with $V_{T}>8 \mathrm{mag}$, in contrast to other star catalogues. It should be remarked that this result is not in contradiction with the statement about the global completeness of Tycho-2 in Høg et al. (2000). However, for studies in small excerpts of the Tycho-2 data, one has to be aware of these regional variations in the completeness. On the other hand, the quality of the Tycho-2 proper motions appears to be as reliable as the Hipparcos data.

Regarding upcoming missions of astrometry satellites - FAME (Horner et al. 1998), DIVA (Röser et al. 1998a), or GAIA (Lindegren \& Perryman 1998) - studies based on uniform all-sky data samples like the IMF investigations demonstrated in this work will play a more important role in the future: The limiting magnitudes of these instruments will be fainter, proper motions will be available for all detected objects (in contrast to, e.g., the Hipparcos catalogue, the proper motions of which were determined for the stars of an input catalogue only) and will become independent of ground based observations, so that their 
data quality will be higher. This means that - concerning the determination of mass functions - the IMF of nearby clusters will be covered down to lower masses. Due to the fainter limiting magnitude and the higher accuracy of the proper motions (and hence a more precise membership determination) a larger number of open star clusters can then be studied.

Acknowledgements. The authors acknowledge Santi Cassisi for providing the isochrones necessary for our studies. For valuable discussions, we thank Andrea Dieball (especially for the hints concerning the differential reddening and the study of the stellar density) and Ram Sagar. Thanks to Klaas S. de Boer and Andrea Dieball for carefully reading the manuscript of this publication. This research has made use of NASA's Astrophysics Data System Bibliographic Services, the CDS data archive in Strasbourg, France, and J.-C. Mermilliod's WEBDA database of open star clusters.

\section{References}

Ahumada, J., \& Lapasset, E. 1995, A\&AS, 109, 375

Baumgardt, H. 1998, A\&A, 340, 402

Bono, G., Caputo, F., Cassisi, S., Castellani, V., \& Marconi, M. 1997, ApJ, 479, 279

Carrier, F., Burki, G., \& Richard, C. 1999, A\&A, 341, 469

Credner, T. 1996, Diploma Thesis, University of Bonn

Eggen, O. J. 1970, ApJ, 161, 159

Eggen, O. J. 1983, AJ, 88, 197

Eggen, O. J. 1986, AJ, 92, 1074

Eichhorn, H. 1974, Astronomy of star positions (Frederick Ungar Publishing, New York)

ESA 1997, The Hipparcos and Tycho Catalogues, ESA SP1200

Foster, D. C., Theissen, A., Butler, C. J., et al. 2000, A\&AS, 143,409

Fresneau, A. 1980, AJ, 85, 66

Hambly, N. C., Hodgkin, S. T., Cossburn, M. R., \& Jameson, R. F. 1999, MNRAS, 303, 835

Høg, E., Kuzmin, A., Bastian, U., et al. 1998, A\&A, 335, L65

Høg, E., Fabricius, C., Makarov, V. V., et al. 2000, A\&A, 355, L27

Horner, S. D., Germain, M. E., Greene, T. P., et al. 1998, BAAS, 193, 1206

Jones, B. F., \& Cudworth, K. 1983, AJ, 88, 215

Jones, B. F., \& Stauffer, J. R. 1991, AJ, 102, 1080

Kraft, R. P. 1967, ApJ, 148, 129

Lindegren, L., \& Perryman, M. A. C. 1998, Highlights in Astr., 11,581

Lyngå, G. 1987, Catalog of open cluster data, 5th edition

Manteiga, M., Martinez-Roger, C., Morales, C., \& Sabau, L. 1991, A\&AS, 87, 419

Makarov, V. V., Odenkirchen, M., \& Urban, S. 2000, A\&A, 358,923

Meusinger, H., Schilbach, E., \& Souchay, J. 1996, A\&A, 312, 833

Mitchell, R. I. 1960, ApJ, 132, 68

Mohan, V., \& Sagar, R. 1985, MNRAS, 213, 337

Monet, D., Bird, A., Canzian, B., et al. 1998, U.S. Naval Observatory Flagstaff Station and Universities Space
Research Association

Narayanan, V. K., \& Gould, A. 1999, ApJ, 523, 328

Nicolet, B. 1981, A\&A, 104, 185

Pandey, A. K., Bhatt, B. C., Mahra, H. S., \& Sagar, R. 1989, MNRAS, 236, 263

Perry, C. L., \& Hill, G. 1969, AJ, 74, 899

Petrie, R. M., \& Heard, J. F. 1969, Pub. Dom. Ap. Obs. Victoria, 13, 329

Pinfield, D. J., Hodgkin, S. T., Jameson, R. F., \& Cossburn, M. R. 1999, IrAJ, 26, 94

Pinsonneault, M. H., Stauffer, J., Soderblom, D. R., King, J. R., \& Hanson, R. B. 1998, ApJ, 504, 170

Piskunov, A. 1980, Bull. Inf. Cent. Données Stellaires, 19, 67

Platais, I., Kozhurina-Platais, V., \& van Leeuwen, F. 1998, AJ, 116, 2423

Prosser, C. F. 1992, AJ, 103, 488

Prosser, C. F. 1994, AJ, 107, 1422

Raboud, D., \& Mermilliod, J.-C. 1998, A\&A, 333, 897

Rachford, B. L., \& Canterna, R. 2000, AJ, 119, 1296

Robichon, N., Arenou, F., Mermilliod, J.-C., \& Turon, C. 1999, A\&A, 345, 471

Röser, S., \& Bastian, U. 1994, A\&A, 285, 875

Röser, S., Bastian, U., de Boer, K. S., et al. 1998, Highlights in Astr., 11, 583

Röser, S., Morrison, J., Bucciarelli, B., Lasker, B., \& McLean, B. 1998b, Contents, Test Results, and Data Availability for GSC 1.2, in Proc. IAU Symp. 179, New Horizons from Multi-Wavelength Sky Surveys, ed. B. McLean, D. A. Golombek, J. J. E. Hayes, \& H. E. Payne (Kluwer, Dordrecht), 420

Salpeter, E. E. 1955, ApJ, 121, 161

Sanders, W. L. 1971, A\&A, 14, 226

Sanner, J., Altmann, M., Brunzendorf, J., \& Geffert, M. 2000, A\&A, 357, 471

Scalo, J. M. 1986, Fund. Cosm. Phys., 11, 1

Scalo, J. M. 1998, The IMF revisited - A case for variations, in ASP Conf. Ser. 142 , ed. G. Gilmore, \& D. Howell (ASP, San Francisco), 201

Schaller, G., Schaerer, D., Meynet, G., \& Maeder, A. 1992, A\&AS, 96, 269

Slettebak, A. 1985, ApJS, 59, 769

Strobel, A. 1991, AN, 312, 177

Tarrab, I. 1982, A\&A, 109, 285

Tsujimoto, T., Yoshii, Y., Nomoto, K., et al. 1997, ApJ, 483, 228

Urban, S. E., Corbin, T. E., \& Wycoff, G. L. 1998, AJ, 115, 2161

van Leeuwen, F. 1980, Mass and luminosity function of the Pleiades, in IAU Symp. 85, ed. J. E. Hesser (Reidel, Dordrecht), 157

van Leeuwen, F. 1999a, A\&A, 341, L71 (vL99a)

van Leeuwen, F. 1999b, Open Cluster Distances from HIPPARCOS parallaxes, in ASP Conf. Ser. 167, ed. Egret D., \& Heck A. (ASP, San Francisco), 52

Westerlund, B. E., Lundgren, K., Pettersson, B., Garnier, R., \& Breysacher, J. 1988, A\&AS, 76, 101

Williams, D. M., Rieke, G. H., \& Stauffer, J. R. 1995, ApJ, 445,359

Wood, H. 1970, The Astrographic Catalogue as a source of old epoch places, in IAU Colloq. 7: Proper Motions, ed. W. J. Luyten (University of Minnesota, Minneapolis), 67 